\title{
Dor musculoesquelética idiopática difusa na infância e na adolescência
}

\author{
Diffuse idiopathic musculoskeletal pain in childhood and adolescence
}

\author{
Juliana Molina ${ }^{1}$, Simone Guerra L. Silva ${ }^{1}$, Fernanda Moreira Teles ${ }^{1}$, Melissa Mariti Fraga ${ }^{2}$, Luciana Tudech S. P. Paulo², Vanessa \\ Bugni², Maria Teresa R. A. Terreri', Maria Odete E. Hilário4, Claudio Arnaldo Len³
}

\section{RESUMO}

Objetivo: A dor musculoesquelética difusa idiopática persistente ou intermitente é definida pela presença de dor com duração de três meses ou mais, sem etiologia aparente, e tem acometido cada vez mais crianças e adolescentes. Este estudo objetivou relatar dois pacientes com dor incapacitante submetidos à abordagem e ao tratamento multiprofissional, além de discutir o tratamento conduzido, por meio de revisão da literatura atual.

Descrição do caso: O primeiro caso é de uma menina com nove anos de idade, com histórico de cefaleia, dor musculoesquelética e dor abdominal há dois anos, período no qual apresentou quatro episódios de ausência de marcha, sem causas orgânicas aparentes. O segundo caso refere-se a uma adolescente de 14 anos com lombalgia diária há 14 meses de forte intensidade, com irradiação para membros inferiores, acompanhada de cefaleia, fadiga e adinamia. Ambas apresentavam exame físico normal, exceto pela presença de alodínia (caso 1) e pontos de fibromialgia (caso 2); além de exames subsidiários normais, sem justificativas orgânicas para as queixas dolorosas. Houve melhora clínica significativa após diagnóstico e tratamento diferencial por meio de abordagem multiprofissional (médico, psicólogo, fisioterapeuta e nutricionista), com retomada das atividades regulares pelas pacientes.

Comentários: A atuação integrada da equipe em um curto período de tempo proporcionou às pacientes a retomada de suas atividades normais, visto que fatores emocionais, físicos e nutricionais relacionam-se à dor.

Palavras-chave: dor; fibromialgia; criança; adolescente.

\section{ABSTRACT}

Objective: Persistent or intermittent diffuse idiopathic musculoskeletal pain is defined by the presence of pain over three months with no apparent etiology. The diagnosis of this condition in children and adolescents is increasing. The objective of this study was to report the cases of two patients with disabling pain that received multidisciplinary treatment and to review the current literature about this subject.

Case description: The first patient is a nine-year old girl with history of headache, musculoskeletal pain and abdominal pain for two years. During this period she had four episodes of absence of gait with no apparent organic cause. The second patient is a 14-year girl with severe daily low back pain for 14 months, followed by headache and fatigue. Both patients presented a normal physical examination, except for allodynia (case 1) and fibromyalgia tender points (case 2). All tests resulted negative regarding the search for organic reasons for pain complaints. Significant clinical improvement was observed with a multidisciplinary approach offered by pediatricians, psychologists, physical therapists, and nutritionists. Both patients resumed their daily regular activities.

Comments: Team integrated performance in a short-term period can provide the attenuation of pain symptoms since emotional, physical and nutritional factors are related to the pain expressed by the patients.

Key-words: pain; fibromyalgia; child; adolescent.
Instituição: Ambulatório de Dor do Setor de Reumatologia da Disciplina de Alergia, Imunologia e Reumatologia do Departamento de Pediatria da Universidade Federal de São Paulo (Unifesp), São Paulo, SP, Brasil

'Mestre em Ciências pelo Programa de Pós-Graduação de Ciências Aplicadas à Pediatria da Unifesp, São Paulo, SP, Brasil

${ }^{2}$ Pós-Graduanda do Setor de Reumatologia do Departamento de Pediatria da Unifesp; Médica do Setor de Reumatologia do Departamento de Pediatria da Unifesp, São Paulo, SP, Brasil

${ }^{3}$ Doutor em Pediatria pela Unifesp; Professor Adjunto do Setor de Reumatologia do Departamento de Pediatria da Unifesp, São Paulo, SP, Brasil ${ }^{4}$ Livre-Docente em Pediatria pela Unifesp; Professora Associada e Chefe do Setor de Reumatologia do Departamento de Pediatria da Unifesp, São Paulo, SP, Brasil
Endereço para correspondência:

Claudio Arnaldo Len

Rua Borges Lagoa, 802 - Vila Clementino

CEP 04038-002 - São Paulo/SP

E-mail: len.cl@terra.com.br

Conflito de interesse: nada a declarar

Recebido em: 22/3/2010

Aprovado em: 20/9/2010 


\section{Introdução}

A dor em membros é comum na faixa etária pediátrica. Cerca de 10 a $20 \%$ das crianças aparentemente saudáveis queixam-se desse sintoma. Na maioria dos pacientes, são casos leves, transitórios e que não alteram a vida cotidiana. No entanto, em algumas situações, a dor é de forte intensidade e tem impacto na qualidade de vida, nos aspectos físicos, emocionais, sociais e escolares ${ }^{(1)}$.

A queixa de dor persistente ou intermitente com duração de três meses ou mais define uma entidade denominada "dor musculoesquelética idiopática” (DMEI). Caracterizada pela amplificação da dor sem uma etiologia aparente ${ }^{(1)}$, esta pode apresentar-se de forma difusa, profunda e bilateral, com duração, periodicidade e intensidade variáveis. É mais frequente em membros inferiores e extra-articular, principalmente em região anterior de pernas e coxas, região poplítea e panturrilhas; contudo pode acometer diferentes regiões do corpo e, na maioria das vezes, é intensa, fugaz, vespertina ou noturna e costuma ocorrer após exercício físico.

A DMEI pertence ao grupo das síndromes de amplificação dolorosa, grupo no qual também se incluem fibromialgia, síndromes complexas regionais e dor idiopática localizada, sendo que cada subgrupo apresenta critérios de classificação bem estabelecidos ${ }^{(1,2)}$. A prevalência da DMEI na faixa etária pediátrica varia significativamente entre 1 até $15 \%^{(3)}$. A maioria dos casos envolve crianças no final da infância e na adolescência, com média de idade de início dos sintomas entre 12 e 13 anos, prevalecendo o sexo feminino ${ }^{(1)}$.

Ainda que de etiologia desconhecida, é provável que a causa da DMEI seja multifatorial, com a contribuição de fatores intrínsecos (baixo limiar para a dor, resposta peculiar no sistema nervoso central, presença de hipermobilidade articular, estresse e funcionamento psíquico) e extrínsecos (experiências dolorosas prévias, privação social, abuso físico e sexual, modelos parentais de comportamento doloroso, distúrbios do sono e baixo condicionamento físico $)^{(4)}$. A importância relativa desses fatores varia entre os indivíduos e o grau de influência dos diferentes fatores também podem se modificar ao longo do tempo ${ }^{(5)}$. Devido a dificuldades ainda existentes na realização de um diagnóstico assertivo, pacientes com DMEI comumente possuem um longo histórico de exames subsidiários, tratamentos médicos e até mesmo internações, com poucos resultados na melhora do quadro doloroso.

Nesse contexto, o objetivo deste estudo foi relatar os casos de dois pacientes com DMEI, bem como discutir a conduta realizada, sendo o relato aprovado pelo Comitê de Ética em Pesquisa da Universidade Federal de São Paulo (Unifesp).

\section{Descrição do caso}

Nos últimos dois anos foram atendidas 110 crianças $\mathrm{e}$ adolescentes com dor musculoesquelética no Ambulatório de Dor do Setor de Reumatologia Pediátrica da Escola Paulista de Medicina da Unifesp, todas encaminhadas da triagem pediátrica ou de serviços da rede pública do Estado de São Paulo. Os dois casos selecionados referem-se a pacientes cujo comprometimento significativo da marcha foi a principal queixa pela qual foram encaminhadas ao serviço.

\section{Caso 1}

Trata-se de uma menina de nove anos, procedente de São Paulo, com história de internação pregressa por miosite viral. Atendida em outro serviço médico, descartou-se a suspeita de doença muscular inflamatória por meio de exames voltados para a pesquisa de inflamações musculares (enzimas). Há dois anos iniciou quadro de dores abdominais e cefaleia recorrentes com duração de aproximadamente 15 dias, com fraqueza e sonolência. A mãe relata que a filha acordou com dor nos membros inferiores e as pernas frias, sem conseguir manterse em pé e andar; esse quadro persistiu por 21 dias. Sem diagnóstico, a paciente retornou a andar espontaneamente. Continuou em investigação neurológica, sempre apresentando as mesmas queixas, sem lesões aparentes. Há um ano, parou subitamente a deambulação por 17 dias, também com retorno espontâneo da marcha, mas apresentando dificuldades e dor intermitente mesmo em repouso. No total, apresentou quatro episódios de perda de marcha. Foi encaminhada para o serviço da Unifesp há seis meses, com dores difusas pelo corpo, principalmente em membros inferiores, e ausência de marcha há dois meses, sem conseguir manter-se em pé. Referia uso de amitripitilina $25 \mathrm{mg}$ há um mês.

O exame clínico foi normal, exceto pela presença de dor em resposta à palpação de fraca intensidade na face anterior das coxas (alodínia). Não foram detectados hipermobilidade articular ou pontos de fibromialgia. Foram solicitados os seguintes exames laboratoriais: hemograma, provas de atividade inflamatória, fator antinúcleo, função tireoidiana, desidrogenase lática, creatinoquinase e aldolase. Diante de resultados normais, foi proposta a hipótese diagnóstica de DMEI. A paciente foi avaliada pela equipe multidisciplinar que fez os seguintes pareceres: 
- Psicologia: os quadros dolorosos mais graves com perda da marcha eram sempre precedidos por episódios nos quais vivenciava algum tipo de conflito familiar ou frente a situações nas quais se sentia desamparada, demonstrando elevados níveis de ansiedade, temores relacionados à ausência da mãe e no convívio social (principalmente escolar), sentimentos de rejeição e baixo limiar à frustração. Foi iniciada psicoterapia semanal.

- Nutrição: paciente com os seguintes índices antropométricos: P/E 103,5\%, E/I 102,5\%; diagnóstico nutricional de eutrofia. Mantinha alimentação balanceada, porém com baixo consumo de legumes e verduras. Nos dias em que apresentava mais dores e referia estados ansiosos, diminuía o consumo alimentar e se alimentava apenas de "beliscos". Foi orientada a modificar o hábito alimentar de forma gradativa, visando à aderência ao tratamento.

- Reabilitação: a principal disfunção observada foi o comprometimento grave da marcha, com dependência para o deslocamento. A criança chegava ao atendimento no colo da mãe e segundo relato desta, a criança arrastava-se para realizar deslocamentos. Demonstrou resistência ao tratamento. Foram utilizadas técnicas de dessensibilização dos membros inferiores, massagens, alongamentos, orientação aos pais e descarga de peso.

Após dez meses de acompanhamento, com melhora do quadro geral e recuperação da marcha com movimentos compensatórios, sem alterações musculoesqueléticas residuais, a paciente apresentou novamente perda da marcha, após um episódio familiar no qual mostrou altos níveis de ansiedade. Foi encaminhada para avaliação psiquiátrica, sendo aumentada a dose de amitripitilina para $125 \mathrm{mg} /$ dia. A reabilitação foi reiniciada, com foco maior nos atendimentos fisioterápicos, visando ao não comprometimento dos membros inferiores, e no acompanhamento psicológico, com o intuito de dar suporte necessário para identificar e controlar os agentes desencadeadores de crises de ansiedade. Houve recuperação da marcha e diminuição de queixas dolorosas em 40 dias e a paciente se manteve assintomática (há 12 meses sem queixas).

\section{Caso 2}

O Caso 2 é o de uma adolescente de 14 anos, sexo feminino, procedente de São Bernardo do Campo (SP). Há 18 meses iniciou quadro de lombalgia diária, de forte intensidade, com irradiação para membros inferiores.
Acompanhava cefaleia, em regiões frontais e temporais, chegando a despertar a noite com dor nas costas. Relatou que na época do surgimento dos sintomas procurou um ortopedista, recebendo diagnóstico de escoliose. Foi prescrito tratamento fisioterápico por dois meses, sem melhora. Há um ano houve piora da dor. Depois de uma melhora espontânea por 30 dias, o quadro álgico se intensificou, resultando na perda temporária da marcha. Em janeiro de 2009, relatou novamente piora do quadro de cefaleia. Retornou ao ortopedista que introduziu anti-inflamatório não esteroidal e miorrelaxantes, sem resposta adequada.

A adolescente procurou o serviço com queixas de dor difusa, fadiga e adinamia. No exame físico, apresentava bom estado geral e dor lombar. Havia dor à palpação em 16/18 pontos de fibromialgia. Todos os exames subsidiários eram normais, incluindo ressonância magnética de coluna lombar e tomografia de crânio recentes. Os achados da equipe interdisciplinar estão relacionados a seguir:

- Psicologia: a paciente apresentava elevado nível de ansiedade de modo a comprometer o funcionamento de comportamentos adaptativos frente a situações de estresse.

- Nutrição: a paciente apresentava sobrepeso, com índice de massa corpórea de 23,84kg/m² (percentil 85). Referiu consumo alimentar acima do recomendado, principalmente nos dias em que apresentava dores, com ingestão excessiva de alimentos fontes de açúcares e gorduras e baixo consumo de legumes e verduras.

- Reabilitação: observado comprometimento na marcha independente, necessitando de apoio para andar. No tratamento, foram utilizadas técnicas de dessensibilização dos membros inferiores, massagens, alongamentos, orientação à paciente e à mãe. A adolescente aderiu ao tratamento, com uma sessão semanal, e realizou exercícios de alongamento, além de caminhadas diárias após a melhora da marcha. Houve retorno da marcha independente, sem sequelas, na quarta semana de acompanhamento.

Depois de oito meses do início do acompanhamento, a adolescente apresentou ausência do quadro álgico e significativa melhora na qualidade vida, adquirida com a incorporação de melhores hábitos alimentares, prática frequente de atividade física (natação semanal e caminhadas diárias), acupuntura e psicoterapia cognitiva comportamental. Os reflexos dessa melhora podem ser observados no relato da jovem e também na ausência de limitações em suas atividades rotineiras (escola e passeios, antes comprometidos pelas dores) e no retorno à prática de atividades diárias. 


\section{Discussão}

A DMEI é uma patologia pouco reconhecida na prática clínica diária, o que faz com que muitas crianças e adolescentes procurem vários médicos e realizem uma série de exames subsidiários desnecessários para afastar doenças ortopédicas, neurológicas e reumatológicas. Em muitos casos, há demora no diagnóstico e na elaboração de um plano de tratamento individual, baseado na abordagem multiprofissional, o que faz com que os pacientes permaneçam por longos períodos sofrendo com sintomas e acabem reduzindo suas atividades cotidianas.

$\mathrm{Na}$ maioria dos pacientes, a dor é de caráter intermitente, forte intensidade e os quatro quadrantes do corpo são acometidos. Achados clínicos comuns são: hipermobilidade articular, alodínia e hiperalgesia à palpação dos membros. Outras manifestações são fadiga, cefaleia, síndrome do colo irritável e distúrbios de sono (insônia, sono não restaurador e sonolência diurna) ${ }^{(6)}$, queixas estas observadas em ambas as pacientes. Ainda, a segunda paciente apresentou 16/18 pontos dolorosos e preencheu critérios para fibromialgia ${ }^{(7)}$. No Serviço de Reumatologia da Unifesp, cerca de 30\% dos pacientes com dor musculoesquelética preenchem esses critérios $^{(8)}$, que, apesar de ainda não terem sido validados para crianças, são utilizados na prática clínica diária.

A prevalência da fibromialgia em crianças e adolescentes saudáveis é de 1 a $6 \%{ }^{(9,10)}$; no entanto, esses dados são subestimados, uma vez que a pesquisa dos pontos dolorosos e o interrogatório sobre o padrão de sono não são rotineiramente realizados nos consultórios pediátricos. Ainda no interrogatório, deve ser avaliada a presença de dor crônica e/ou recorrente em outros membros da família; em estudo realizado na Unifesp (dados não publicados), 70\% das mães de adolescentes com fibromialgia preencheram critérios diagnósticos para a doença, especulando-se que essa relação possa se associar a uma base genética e a fatores ambientais.

A etiopatogenia da DMEI é multifatorial, e traumas físicos e/ou emocionais podem ser gatilhos; uma anamnese detalhada com pais e pacientes pode ser reveladora de situações como abuso psicológico e até mesmo sexual ${ }^{(1)}$. A dor é, então, perpetuada e retroalimentada por situações associadas à ansiedade, ao medo e à depressão, bem como a uma "desregulação" dos hábitos de vida, como alimentação inadequada, relação sono-vigília, redução do nível das atividades físicas, sociais e escolares ${ }^{(4)}$. Nos dois casos relatados, as pacientes apresentavam ansiedade, estresse e problemas de autoestima relacionados à amplificação da dor.
Do ponto de vista psicológico, os aspectos emocionais e as características de personalidade podem estabelecer relações diretas com a gênese da dor sem causa orgânica. Algumas dessas características podem se associar ao aumento da suscetibilidade à dor, podendo ser a dor também sintoma de doenças mentais, como ansiedade generalizada e depressão. Estudos realizados por meio de comparações entre grupos de adolescentes com fibromialgia e seus familiares e com adolescentes e familiares sem queixas de dor verificaram que mães do primeiro grupo relatam o dobro de queixas dolorosas e maior frequência de sintomas depressivos do que as mães dos pares de comparação. O grupo com fibromialgia também demonstrou relações familiares mais conflituosas e esse histórico de queixas dolorosas em mães de adolescentes com dor foi associado pelos autores a maior prejuízo funcional ${ }^{(11)}$.

No ambulatório da Unifesp, todos os pacientes com dor são avaliados por psicólogos e, havendo necessidade, acompanhados em sessões semanais. As pacientes descritas nesse estudo demonstravam elevados níveis de ansiedade e preocupações, com pobreza de repertório adequado frente a situações interpretadas como de desamparo. Em ambos os casos, as situações ansiogênicas estavam diretamente relacionadas à ausência da mãe. No entanto, cabe esclarecer que, nestes casos, ausências rotineiras das mães, como ida ao trabalho e às compras, eram ocasionalmente desencadeadoras de ansiedade.

Segundo Graham e Streitel ${ }^{(12)}$, indivíduos com dor crônica têm maior ocorrência de perturbações comportamentais. A explicação para isso se daria em razão a uma interpretação aumentada de estressores psicológicos em diversos aspectos de suas vidas quando comparados a indivíduos sem dor crônica. A associação entre humor deprimido, estresse, dor e sono é suscetível de ser particularmente forte em indivíduos jovens, que ainda estão desenvolvendo suas habilidades para regular a emoção, a atenção e as respostas comportamentais ao estresse $^{(13)}$. Dessa forma, tais estudos sugerem que múltiplos estressores podem aumentar o risco de problemas comportamentais e de saúde.

As pacientes aqui relatadas apresentaram diminuição da capacidade funcional, com limitação da deambulação. Em pacientes com DMEI, a disfunção da marcha e o quadro doloroso são de importância relevante, levando a prejuízos na qualidade de vida de crianças e adolescentes e gerando comprometimento nas atividades de vida diária, sociais e elevado número de faltas escolares. A inatividade física tende a se agravar ao longo dos meses; em muitas situações, os 
pacientes são orientados a restringir exercícios com o objetivo de reduzir a dor. Mas, no caso da DMEI, essa conduta passa a contribuir para o agravo da dor, além de diminuir a massa muscular e piorar as alterações posturais ${ }^{(14)}$.

O tratamento de crianças com DMEI é baseado na relação médico-paciente e no suporte multiprofissional. Muitos pais, frente à ausência de um diagnóstico assertivo e esclarecedor das queixas dolorosas de seus filhos, chegam a duvidar da a veracidade dessas queixas. O clínico deve reforçar que a dor existe, é física e que muitos pacientes apresentam limiar para a dor reduzido. A escuta dessas queixas, os esclarecimentos e a validação do sofrimento do paciente com DMEI são o foco inicial no atendimento psicoterápico. Desse modo, o esquema terapêutico ideal envolve reabilitação, com ênfase nos exercícios físicos aeróbicos, psicoterapia, além de outras modalidades, como a acupuntura ${ }^{(15)}$.

A utilização de técnicas como relaxamento, reeducação postural, cinesioterapia e meios físicos (massoterapia, hidroterapia, frio, calor, estimulação elétrica, entre outros) proporciona alívio sintomático da dor, possibilitando a redução do uso de medicamentos ${ }^{(16)}$. Em estudo piloto de pacientes entre oito e 18 anos, randomizados para uma intervenção com 12 semanas de exercício com sessões de treino semanais, foi demonstrado que crianças com fibromialgia toleram os exercícios aeróbios de intensidade moderada, sem exacerbação da doença e com melhorias significativas na função física ${ }^{(17)}$. Outros estudos indicam que o tratamento com exercício físico orientado, quando comparado com um grupo controle sem exercício, pode ter efeitos positivos na redução da dor ${ }^{(17)}$.

Revisões sistemáticas vem sendo feitas com o intuito de comprovar a eficácia de terapias psicológicas para o manejo da dor crônica e recorrente em crianças. Técnicas como relaxamento, hipnose, treinamento de habilidades, biofeeback e terapia cognitiva comportamental são hoje reconhecidas como intervenções que podem ajudar no controle das dores, evitando suas consequências incapacitantes. No caso de crianças e jovens, existem também boas evidências da eficácia na redução da gravidade e frequência da dor de cabeça crônica, dor abdominal recorrente e fibromialgia, com efeitos duradouros ${ }^{(18)}$.

A prevalência da obesidade em crianças e adolescentes tem aumentado em diversos países do mundo, inclusive no Brasil, o que está fortemente relacionado a mudanças no estilo de vida e nos hábitos alimentares, como o fácil acesso e o baixo custo de alimentos ricos em gorduras e açúcares ${ }^{(19)}$.
$\mathrm{Na}$ infância, além de a criança exercer pouco controle sobre a disponibilidade domiciliar de alimentos, pode sofrer influência do hábito alimentar e de atividade física dos pais e familiares ${ }^{(20)}$. Em ambos os caso apresentados, as pacientes mantinham um hábito alimentar inadequado, decorrente da influência e dos costumes da família. Wright et al, em estudo transversal, avaliaram 3.471 gêmeos americanos com idade média de 31 anos. A prevalência de sobrepeso e obesidade foi de 24,3 e $13,3 \%$, respectivamente. Entre os indivíduos com queixa de dor, $1,8 \%$ apresentavam fibromialgia, sendo esse diagnóstico encontrado em maior proporção em pacientes com excesso de peso $^{(21)}$, o que significa que o peso deve ser controlado e os hábitos alimentares adequados precisam ser estimulados desde a infância.

Segundo revisão realizada em 2009 por Häuser $e t$ al em adultos, a utilização de medicações antidepressivas está associada à melhoria de dor, depressão, fatiga, distúrbios do sono e qualidade de vida nos pacientes com fibromialgia ${ }^{(22)}$. No entanto, Buskila, em 2009, sugeriu que o tratamento de crianças e adolescentes fosse iniciado preferencialmente por modalidades não farmacológicas, incluindo reeducação, prática de exercícios e acompanhamento psicológico, visto não existirem estudos sistemáticos sobre o uso de medicamentos de crianças ${ }^{(23)}$ Em nosso ambulatório, são prescritos medicamentos, como os antidrepressivos tricíclicos, apenas nos casos em que não há melhora com as modalidades de tratamento não farmacológicas. Busch et al compararam 175 pacientes com fibromilagia, dos quais 87 receberam apenas amitriptilina e 88 realizaram apenas fisioterapia. Aplicando o Questionário de Impacto da Fibromialgia (FIQ), observouse melhora com a fisioterapia. Tanto a alta pontuação no FIQ na linha de base quanto o baixo nível socioeconômico foram preditores significativos do benefício de ambos os tratamentos. Os autores concluíram que a terapia isolada com amitriptilina ou com fisioterapia foi igualmente eficaz, durante período de seis meses ${ }^{(24)}$

Em conclusão, pacientes com DMEI podem apresentar diminuição da capacidade funcional significativa e, em alguns casos, ocorre comprometimento da marcha sem causa física aparente. $\mathrm{Na}$ investigação diagnóstica, é necessário excluir as doenças orgânicas, mas também é importante uma avaliação pela equipe multiprofissional, uma vez que aspectos emocionais, físicos e nutricionais estão presentes. $\mathrm{O}$ tratamento deve ser planejado com base na experiência dos diversos profissionais envolvidos. 


\section{Referências bibliográficas}

1. Malleson PN, al-Matar M, Petty RE. Idiopathic musculoskeletal pain syndromes in children. J Rheumatol 1992;19:1786-9.

2. Merskey $\mathrm{H}$, Bogduk $\mathrm{N}$. Classification of chronic pain: descriptions of chronic pain syndromes and definitions of pain terms. Seatle: IASP Press; 1994.

3. Goodman JE, McGrath PJ. The epidemiology of pain in children and adolescents: a review. Pain 1991;46:247-64.

4. Malleson P, Clinch J. Pain syndromes in children. Curr Opin Rheumatol 2003;15:572-80.

5. Sherry DD, Malleson PN. The idiopathic musculoskeletal pain syndromes in childhood. Rheum Dis Clin North Am 2002;28:669-85.

6. Oliveira SK. Síndrome de amplificação da dor. In: Knnup S, editor. Reumatologia pediátrica. $2^{\text {nd }}$ ed. Rio de Janeiro: Revinter; 2001. p. 319-28.

7. Wolfe F, Smythe HA, Yunus MB, Bennett RM, Bombardier C, Goldenberg DL et al. The American College of Rheumatology 1990. Criteria for the classification of fibromyalgia. Report of the multicenter criteria committee. Arthritis Rheum 1990;33:160-72.

8. Barbosa CM, Hangai L, Terreri MT, Len CA, Hilário MO. Limb pain in a pediatric rheumatology outpatient clinic. Rev Paul Pediatr 2005;23:63-8.

9. Buskila D, Press J, Gedalia A, Klein M, Neumann L, Boehm R et al. Assessment of non-articular tenderness and prevalence of fibromyalgia in children. $J$ Rheumatol 1993;20:368-70.

10. Roizenblatt S, Hilário MO, Goldenberg J, Tufik S. Fibromialgia juvenil. Rev Bras Reumatol 1997;37:271-4.

11. Kashikar-Zuck S, Lynch AM, Slater S, Graham TB, Swain NF, Noll RB. Family factors, emotional functioning, and functional impairment in juvenile fibromyalgia syndrome. Arthritis Rheum 2008;59:1392-8.

12. Graham JE, Streitel KL. Sleep quality and acute pain severity among young adults with and without chronic pain: the role of biobehavioral factors. J Behav Med 2010;33:335-45.

13. Huntley ED, Campo JV, Dahl RE, Lewin DS. Sleep characteristics of youth with functional abdominal pain and a healthy comparison group. J Pediatr Psychol 2007;32:938-49.

14. Pimenta CA, Teixeira MJ, Simões P, Simões C, Cruz DA, Okada M. League against pain: an undergraduate extracurricular program. Rev Esc Enf USP 1998;32:281-9.

15. Werner R. Guia de patologia para massoterapeutas. $2^{\text {nd }}$ ed. Rio de Janeiro: Guanabara Koogan; 2005.

16. Clay JH, Pounds DM. Massoterapia clínica - integrando anatomia e tratamento. São Paulo: Manole; 2003.

17. Stephens S, Feldman BM, Bradley N, Schneiderman J, Wright V, Singh-Grewal $\mathrm{D}$ et al. Feasibility and effectiveness of an aerobic exercise program in children with fibromyalgia: results of a randomized controlled pilot trial. Arthritis Rheum 2008;59:1399-406.

18. Clinch J, Eccleston C. Chronic musculoskeletal pain in children: assessment and management. Rheumatology (Oxford) 2009;48:466-74.

19. Wang Y, Monteiro C, Popkin BM. Trends of obesity and underweight in older children and adolescents in the United States, Brazil, China, and Russia. Am J Clin Nutr 2002;75:971-7.

20. New South Wales Centre for Public Health Nutrition. Best options for promoting healthy weight and preventing weight gain in NSW. New South Wales: University of Sidney; 2005.

21. Wright LJ, Schur E, Noonan C, Ahumada S, Buchwald D, Afari N. Chronic pain, overweight, and obesity: findings from a community-based twin registry. J Pain 2010;11:628-35.

22. Häuser W, Bernardy K, Uceyler N, Sommer C. Treatment of fibromyalgia syndrome with antidepressants: a meta analysis. JAMA 2009;301:198-209.

23. Buskila D. Pediatric fibromyalgia. Rheum Dis Clin North Am 2009; 35:253-61.

24. Busch A, Schachter CL, Peloso PM, Bombardier C. Exercise for treating fibromyalgia syndrome. Cochrane Database Syst Rev 2007:CD003786. 